\title{
Numerical Computations of Green's Function and Its Fourier Coefficients on $\operatorname{PSL}(2, \mathbb{Z})$
}

\author{
Helen Avelin
}

\section{CONTENTS}

\section{Introduction}

2. Value Statistics for Green's Function

3. Value Statistics for $\boldsymbol{F}_{n}(z ; s)$

4. Further Experiments

Acknowledgments

References
2000 AMS Subject Classification: Primary 11F03; Secondary 11-04, $11 \mathrm{Y} 35$

Keywords: Green's function, resolvent kernel, computational number theory, quantum chaos, Fourier coefficients, spectral theory, pseudo cusp forms, Gaussian value distribution, point scatterer
We present some examples of numerical investigations of the value distribution of Green's function and of its Fourier coefficients on the modular group $\operatorname{PSL}(2, \mathbb{Z})$. Our results indicate that both Green's function $G_{s}(z ; w)$ and its Fourier coefficients $F_{n}(z ; s)$ have a Gaussian value distribution in the semiclassical limit when $\operatorname{Re} s=1 / 2$.

\section{INTRODUCTION}

In [Avelin 10] we developed algorithms for numerical computations of Green's function $G_{s}(z ; w)$ and of its Fourier coefficients $F_{n}(z ; s)$ on Fuchsian groups with one cusp. The algorithms can be used for a variety of numerical investigations of the properties of $G_{s}(z ; w)$ and $F_{n}(z ; s)$. Here we will give some results of a few examples of such investigations.

First recall the basic setting from [Avelin 10]. Let $\Gamma$ be a cofinite Fuchsian group with one cusp. We consider Green's function on a hyperbolic surface of the type $\Gamma \backslash \mathcal{H}$, where $\mathcal{H}$ is the Poincaré upper half-plane equipped with the hyperbolic metric $d s^{2}=y^{-2}\left(d x^{2}+d y^{2}\right)$ and corresponding area $d \mu=d x d y / y^{2}$.

It is known that Green's function (or the resolvent kernel) on $\Gamma \backslash \mathcal{H}$ is an eigenfunction of the Laplace-Beltrami operator

$$
\Delta=y^{2}\left(\frac{\partial^{2}}{\partial x^{2}}+\frac{\partial^{2}}{\partial y^{2}}\right)
$$

with eigenvalue $s(1-s)$; that $G_{s}(z ; w)$ satisfies $G_{s}(z ; w)=G_{s}(w ; z)$; that $G_{s}(z ; w)$ is automorphic in both variables, i.e., $G_{s}(T z ; U w)=G_{s}(z ; w)$ when $T, U \in$ $\Gamma$; and that near $z=w$, we have

$$
G_{s}(z ; w)=\frac{\# \Gamma_{w}}{2 \pi} \ln |z-w|+O(1)
$$

where $\Gamma_{w}$ is the stabilizer of $w$; cf. [Hejhal 83, pp. 3334 (Proposition 6.5)]. For $s$ not a pole, the following Fourier series representation holds for $z=x+i y$ with 
$y>\operatorname{Im} T w$ for all $T \in \Gamma$ (cf. [Hejhal 83, pp. 39-42, 250] and [Avelin 10, Lemma 2.1 (a)]):

$$
\begin{aligned}
G_{s}(z ; w)= & \frac{E(w ; s) y^{1-s}}{1-2 s} \\
& -\sum_{n \neq 0} F_{-n}(w ; s) y^{1 / 2} K_{s-\frac{1}{2}}(2 \pi|n| y) e^{2 \pi i n x} .
\end{aligned}
$$

If $E(w ; s)=0$, then $G_{s}(z ; w)$ is a pseudo cusp form. On $\operatorname{PSL}(2, \mathbb{Z})$, this is the case if, for example, $w=\rho$, where

$$
\rho=\frac{1}{2}+i \frac{\sqrt{3}}{2}
$$

and $s$ is a zero of the Riemann zeta function or a zero of the Dirichlet $L$-function $L\left(s,\left(\frac{-3}{\cdot}\right)\right)$; cf. [Hejhal 81, p. 99, 104-105].

The Fourier coefficients of (1-1) are given by (for $n \neq 0$ and $\operatorname{Re} s>1$ ), cf. [Hejhal 83, p. 41 (6.7)],

$$
\begin{gathered}
F_{n}(z ; s)=\sum_{W_{0} \in \Gamma_{\infty} \backslash \Gamma}\left(\operatorname{Im} W_{0} z\right)^{1 / 2} I_{s-\frac{1}{2}}\left(2 \pi|n| \operatorname{Im} W_{0} z\right) \\
\times e^{2 \pi i n \operatorname{Re} W_{0} z},
\end{gathered}
$$

and $F_{0}(z ; s)=E(z ; s)$, i.e., the Eisenstein series. Each $F_{n}(z ; s)$ has a Fourier series for $z=x+i y$,

$$
\begin{array}{r}
F_{n}(z ; s)= \begin{cases}y^{1 / 2} I_{s-\frac{1}{2}}(2 \pi|n| y) e^{2 \pi i n x}, & n \neq 0, \\
y^{s}, & n=0,\end{cases} \\
+\varphi_{0}^{n}(s) \frac{y^{1-s}}{2 s-1}+\sum_{m \neq 0} \varphi_{m}^{n}(s) y^{1 / 2} K_{s-\frac{1}{2}}(2 \pi|m| y) e^{2 \pi i m x},
\end{array}
$$

valid for $s \in \mathbb{C}, s$ not a pole; cf. [Hejhal 83, pp. 56ff., 254ff.].

The computations in the present paper are restricted to the case $\Gamma=\operatorname{PSL}(2, \mathbb{Z})$. The usual fundamental domain of $\operatorname{PSL}(2, \mathbb{Z})$ (cf. [Hejhal 83, p. 7]) is

$$
\mathcal{F}=\{z \in \mathcal{H}:|z| \geq 1,|\operatorname{Re} z| \leq 1 / 2\} .
$$

We also note that $\rho$, defined in (1-2), is the lower right corner of $\mathcal{F}$.

\section{VALUE STATISTICS FOR GREEN'S FUNCTION}

The $G_{s}(z ; w)$-algorithm described in [Avelin 10] may be used to explore questions in quantum chaology such as those discussed in, for example, [Hejhal and Rackner 92, Hejhal 99, Hejhal and Strömbergsson 01, Avelin 08].

The physical interpretation we have in mind is that some eigenfunctions with singularities correspond to wave functions on a surface with a point scatterer, i.e., a pointlike obstacle situated on the surface, scattering the wave functions. See, for example, [Shigehara and Cheon 97, Shigehara 94], where eigenfunctions in terms of Green's function are used, and [de Vries et al. 98] for a discussion of some physical situations successfully modeled by a point scatterer.

While quantum waves are inevitably influenced by a point scatterer, a classical particle will not even notice the obstacle unless it directly strikes it. For example, a surface that is classically nonchaotic, such as a square, may show chaotic behavior on the quantum level if we introduce a point scatterer. See, for example, the computations in [Šeba 90] on the Sinai billiard with a circular obstacle of radius $r=0$.

Regarding cusp forms and Eisenstein series, we expect that the value distribution on fixed compact subsets approaches a Gaussian distribution in the semiclassical limit, i.e., as the eigenvalue approaches infinity. The experimental results of [Hejhal and Rackner 92, Hejhal 99, Hejhal and Strömbergsson 01, Avelin 08] indeed point in this direction.

Here we show examples of experiments with $G_{s}(z ; w)$ analogous to those done with $E(z ; s)$ in [Avelin 08]. Thus values of $G_{s}(z ; w)$ were computed in a rectangular window

$$
F=[0,0.4] \times[1.6,2.0]
$$

over a $1200 \times 1200$ grid for $\operatorname{Im} s \leq 501$ and a $2500 \times$ 2500 grid for $\operatorname{Im} s$ near 1000. We kept $\operatorname{Re} s=1 / 2$ and used three values of $\operatorname{Im} s$ for which $\zeta(s)=0$, namely 236.524230, 500.309085, and 999.791572, and $w$-values $\rho, i, 0.48+0.88 i, 0.2+1.5 i$, and $0.2+1.8 i$, where only the last one is in $F$. Note that

$$
E(\rho ; s)=0 \quad \text { and } \quad E(i ; s)=0 \quad \text { if } \quad \zeta(s)=0 ;
$$

cf. [Hejhal 92, p. 51] and [Hejhal 81, p. 105].

For readers interested in accuracy and details of our numerical experiments we have gathered some information in Table 1 . Here the notations $M_{0}, Q, r$, and $Y$ are the same as in [Avelin 10, Section 4]. Computations were performed with working precision $P=38$ and precision $P_{B}$ in calls to our $I$ - and $K$-Bessel routines; cf. [Avelin 10, Section 5]. All computations of values of $G_{s}(z ; w)$ used the Fourier expansion (1-1) truncated after $n=M_{0}-2$. The error in the computed values is estimated using

$$
\operatorname{Im} G_{s}(z ; w)=\frac{E(z ; s) \overline{E(w ; s)}}{4 \operatorname{Im} s},
$$

which follows from [Hejhal 83, p. 251, Theorem 3.5(d)], 


\begin{tabular}{|cc|ccccc|}
\hline$w$ & $R$ & $M_{0}$ & $Q$ & $r$ & $P_{B}$ & error \\
\hline \hline$\rho$ & 236.52 & 65 & 80 & 2 & 38 & $1 \mathrm{e}-35$ \\
$\rho$ & 500.31 & 116 & 139 & 2 & 72 & $1 \mathrm{e}-34$ \\
$\rho$ & 999.79 & 213 & 250 & 2 & 154 & $1 \mathrm{e}-17$ \\
\hline$i$ & 236.52 & 79 & 80 & 1 & 38 & $1 \mathrm{e}-35$ \\
$i$ & 500.31 & 138 & 139 & 1 & 72 & $1 \mathrm{e}-33$ \\
$i$ & 999.79 & 249 & 250 & 1 & 154 & $1 \mathrm{e}-33$ \\
\hline $0.48+0.88 i$ & 236.52 & 65 & 80 & 3 & 38 & $1 \mathrm{e}-35$ \\
$0.48+0.88 i$ & 500.31 & 117 & 139 & 3 & 72 & $1 \mathrm{e}-33$ \\
$0.48+0.88 i$ & 999.79 & 214 & 250 & 3 & 154 & $1 \mathrm{e}-31$ \\
\hline $0.2+1.5 i$ & 236.52 & 71 & 80 & 3 & 38 & $1 \mathrm{e}-29$ \\
$0.2+1.5 i$ & 500.31 & 125 & 139 & 3 & 72 & $1 \mathrm{e}-22$ \\
$0.2+1.5 i$ & 999.79 & 229 & 250 & 3 & 154 & $1 \mathrm{e}-20$ \\
\hline $0.2+1.8 i$ & 236.52 & 70 & 80 & 3 & 38 & $1 \mathrm{e}-28$ \\
$0.2+1.8 i$ & 500.31 & 123 & 139 & 3 & 72 & $1 \mathrm{e}-22$ \\
$0.2+1.8 i$ & 999.79 & 225 & 250 & 3 & 154 & $1 \mathrm{e}-21$ \\
\hline
\end{tabular}

TABLE 1. Numerical details of our computations of $G_{\frac{1}{2}+i R}(z ; w)$ on $\operatorname{PSL}(2, \mathbb{Z})$. Our $Y$-values were $0.85,0.86$.

[Avelin 10, Lemma 2.1(a)], and the fact that we have $\operatorname{Re} s=1 / 2$. The maximum of the relative errors at the upper right corner and the lower left corner of $F$ is given in the column marked error. We have noted that the error stays fairly constant over $F$, and so this number may be used as a guide to our overall accuracy. As $\operatorname{Im} z$ approaches $\operatorname{Im} w$ (this happens only when $w \in F$, i.e., in the case $w=0.2+1.8 i)$, the time it takes to compute values using our method tends to infinity. We therefore remove the strip $\mid \operatorname{Im} z-$ $\operatorname{Im} w \mid \leq 0.001$ from our computations (and check that the computational time is acceptable and that the accuracy near this strip is consistent with the estimates in Table 1).

Histograms were made by throwing hyperbolic area into 20 buckets according to the local size of the real and imaginary parts of $G_{s}(z ; w)$. Standard deviation and moments were computed as

$$
\begin{aligned}
\sigma^{2} & =\frac{1}{\mu(F)} \int_{F}\left|\operatorname{Re} G_{s}(z ; w)\right|^{2} d \mu(z), \\
I_{k} & =1-\frac{\frac{1}{\mu(F)} \int_{F}\left|\operatorname{Re} G_{s}(z ; w)\right|^{k} d \mu(z)}{\pi^{-\frac{1}{2}} 2^{\frac{k}{2}} \sigma^{k} \Gamma\left(\frac{k+1}{2}\right)},
\end{aligned}
$$

and similarly for $\operatorname{Im} G_{s}(z ; w)$; thus $I_{k}=0$ for a Gaussian distribution. The integrals giving $\sigma$ and $I_{k}$ in $(2-3)$ were computed as Riemann sums. ${ }^{1}$ The value $\delta$ is the mean difference between the histogram points and the corresponding points on the conjectured Gaussian curve $(\sigma \sqrt{2 \pi})^{-1} \exp \left(-\frac{x^{2}}{2 \sigma^{2}}\right)$.

\footnotetext{
${ }^{1}$ In this regard, note that the grid size $\Delta_{x}$ is about $0.014 \pi / R$, $0.029 \pi / R, 0.028 \pi / R$ (in the hyperbolic metric) for our three respective choices of $s=1 / 2+i R$.
}

We remark that a good fit to a Gaussian curve should not be expected unless the box $F$ is large compared to the de Broglie wavelength, which is related to $\tau=\pi / R$. For our $R$-values (236.524230, 500.309085, and 999.791572), $F$ corresponds to about $17 \tau, 35 \tau$, and $71 \tau$ respectively. Ideally one would like to have a larger $F$. However, this becomes a question of computational time.

The accuracy in our computations of the $I_{k}$ depends on our grid size in $F$ and on how fast $G_{s}(z ; w)$ varies over the grid. We record the discrepancies of $\operatorname{Re} G_{s}(z ; w)$ and $\operatorname{Im} G_{s}(z ; w)$ over the grid and note that these are sometimes as large as $8 \%$ of $\max _{z \in F}\left|\operatorname{Re} G_{s}(z ; w)\right|$ and $\max _{z \in F}\left|\operatorname{Im} G_{s}(z ; w)\right|$, respectively. (In the case with $w=0.2+1.8 i \in F$ it is even worse.) Thus some of our $I_{k}$ are not as accurate as we would have liked. Having a finer grid is, again, a question of computational time. In this (preliminary) experiment it was our priority to study several cases in order to gain an overall understanding. It would be useful to perform a more thorough study of a few of these cases with a larger $F$ and a finer grid size. Our computational time for the data in this paper is on the order of several months.

Our results for $\operatorname{Re} G_{s}(z ; w)$ and $\operatorname{Im} G_{s}(z ; w)$ are shown in Tables 2 and 3 respectively, and in Figures 1, 2, 3 , and 4 . (Note that by $(2-1)$ and $(2-2)$ we have $G_{s}(z ; \rho), G_{s}(z ; i) \in \mathbb{R}$ for our $s$-values.) It is clear that the fit of the values of $\operatorname{Re} G_{s}(z ; w)$ to a Gaussian distribution improves as the eigenvalue increases through our three test values, for all our $w$-cases. For $\operatorname{Im} G_{s}(z ; w)$ (which is much smaller than $\operatorname{Re} G_{s}(z ; w)$ in magnitude) the tendency is not as clear, although the overall picture shows an improvement in $\delta$ and (even more so) the higher moments. 


\begin{tabular}{|cc|cccccc|}
\hline$w$ & $R$ & mean & $\sigma$ & $\delta$ & $I_{4}$ & $I_{6}$ & $I_{8}$ \\
\hline \hline$\rho$ & 236.52 & $-1 \mathrm{e}-03$ & 0.17 & $3 \mathrm{e}-02$ & $8 \mathrm{e}-02$ & $2 \mathrm{e}-01$ & $4 \mathrm{e}-01$ \\
$\rho$ & 500.31 & $1 \mathrm{e}-04$ & 0.16 & $1 \mathrm{e}-02$ & $4 \mathrm{e}-02$ & $1 \mathrm{e}-01$ & $2 \mathrm{e}-01$ \\
$\rho$ & 999.79 & $-1 \mathrm{e}-04$ & 0.48 & $2 \mathrm{e}-03$ & $3 \mathrm{e}-03$ & $1 \mathrm{e}-02$ & $3 \mathrm{e}-02$ \\
\hline$i$ & 236.52 & $1 \mathrm{e}-04$ & 0.12 & $4 \mathrm{e}-02$ & $2 \mathrm{e}-02$ & $1 \mathrm{e}-01$ & $2 \mathrm{e}-01$ \\
$i$ & 500.31 & $1 \mathrm{e}-04$ & 0.19 & $1 \mathrm{e}-02$ & $-3 \mathrm{e}-03$ & $-1 \mathrm{e}-03$ & $2 \mathrm{e}-02$ \\
$i$ & 999.79 & $1 \mathrm{e}-04$ & 0.21 & $5 \mathrm{e}-03$ & $1 \mathrm{e}-02$ & $5 \mathrm{e}-02$ & $1 \mathrm{e}-01$ \\
\hline $0.48+0.88 i$ & 236.52 & $2 \mathrm{e}-02$ & 1.69 & $5 \mathrm{e}-03$ & $7 \mathrm{e}-02$ & $1 \mathrm{e}-01$ & $2 \mathrm{e}-01$ \\
$0.48+0.88 i$ & 500.31 & $-9 \mathrm{e}-05$ & 0.21 & $1 \mathrm{e}-02$ & $4 \mathrm{e}-02$ & $1 \mathrm{e}-01$ & $2 \mathrm{e}-01$ \\
$0.48+0.88 i$ & 999.79 & $-6 \mathrm{e}-05$ & 0.42 & $2 \mathrm{e}-03$ & $4 \mathrm{e}-03$ & $5 \mathrm{e}-03$ & $-2 \mathrm{e}-02$ \\
\hline $0.2+1.5 i$ & 236.52 & $-1 \mathrm{e}-02$ & 0.91 & $1 \mathrm{e}-02$ & $6 \mathrm{e}-02$ & $1 \mathrm{e}-01$ & $3 \mathrm{e}-01$ \\
$0.2+1.5 i$ & 500.31 & $2 \mathrm{e}-04$ & 0.13 & $2 \mathrm{e}-02$ & $-1 \mathrm{e}-02$ & $-2 \mathrm{e}-02$ & $-2 \mathrm{e}-02$ \\
$0.2+1.5 i$ & 999.79 & $6 \mathrm{e}-05$ & 0.15 & $8 \mathrm{e}-03$ & $1 \mathrm{e}-02$ & $3 \mathrm{e}-02$ & $6 \mathrm{e}-02$ \\
\hline $0.2+1.8 i$ & 236.52 & $-2 \mathrm{e}-02$ & 1.63 & $4 \mathrm{e}-03$ & $5 \mathrm{e}-02$ & $1 \mathrm{e}-01$ & $1 \mathrm{e}-01$ \\
$0.2+1.8 i$ & 500.31 & $7 \mathrm{e}-05$ & 0.07 & $2 \mathrm{e}-02$ & $-9 \mathrm{e}-03$ & $-6 \mathrm{e}-02$ & $-1 \mathrm{e}-01$ \\
$0.2+1.8 i$ & 999.79 & $5 \mathrm{e}-05$ & 0.28 & $5 \mathrm{e}-03$ & $4 \mathrm{e}-03$ & $1 \mathrm{e}-02$ & $7 \mathrm{e}-03$ \\
\hline
\end{tabular}

TABLE 2. Value statistics of $\operatorname{Re} G_{\frac{1}{2}+i R}(z ; w)$ on $\operatorname{PSL}(2, \mathbb{Z})$ in the box $F$.
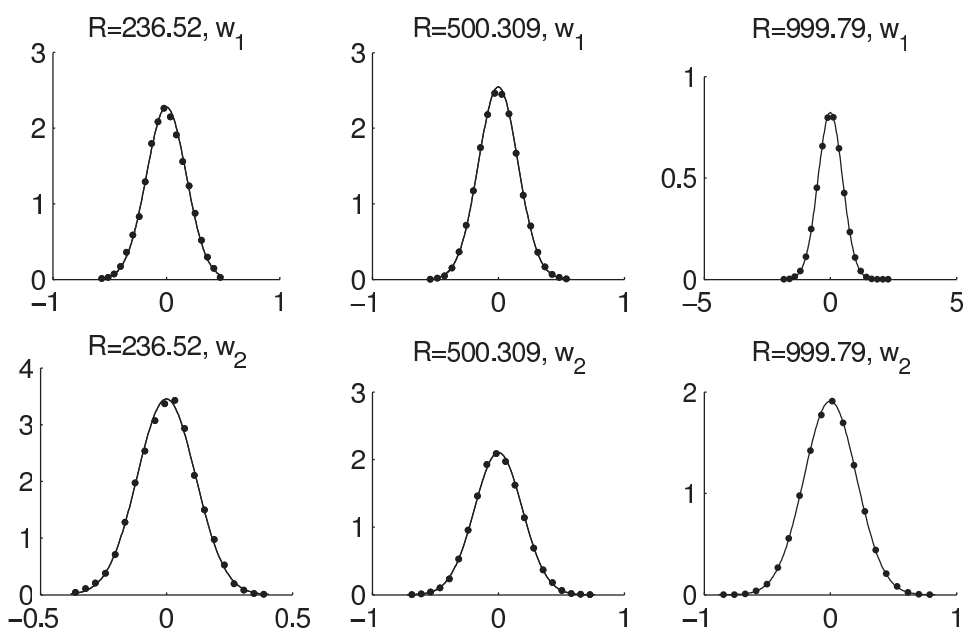

FIGURE 1. Histograms of the real-valued $G_{\frac{1}{2}+i R}(z ; w)$ with $w_{1}=\rho$ and $w_{2}=i$. The solid lines are Gaussian curves.
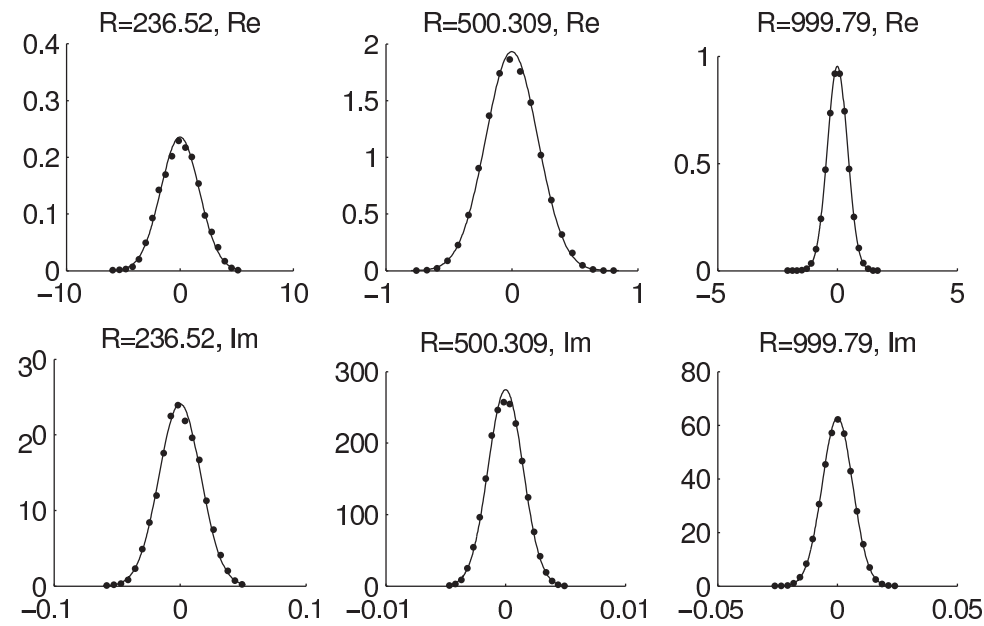

FIGURE 2. Histograms of the real and imaginary parts of $G_{\frac{1}{2}+i R}(z ; 0.48+0.88 i)$, in the first and second rows respectively. The solid lines are Gaussian curves. 


\begin{tabular}{|cc|cccccc|}
\hline$n$ & $R$ & mean & $\sigma$ & $\delta$ & $I_{4}$ & $I_{6}$ & $I_{8}$ \\
\hline \hline $0.48+0.88 i$ & 236.52 & $2 \mathrm{e}-04$ & 0.017 & $3 \mathrm{e}-01$ & $6 \mathrm{e}-02$ & $1 \mathrm{e}-01$ & $3 \mathrm{e}-01$ \\
$0.48+0.88 i$ & 500.31 & $-1 \mathrm{e}-05$ & 0.002 & $4 \mathrm{e}+00$ & $8 \mathrm{e}-02$ & $2 \mathrm{e}-01$ & $3 \mathrm{e}-01$ \\
$0.48+0.88 i$ & 999.79 & $-2 \mathrm{e}-05$ & 0.006 & $2 \mathrm{e}-01$ & $2 \mathrm{e}-02$ & $7 \mathrm{e}-02$ & $1 \mathrm{e}-01$ \\
\hline $0.2+1.5 i$ & 236.52 & $3 \mathrm{e}-04$ & 0.021 & $3 \mathrm{e}-01$ & $6 \mathrm{e}-02$ & $1 \mathrm{e}-01$ & $3 \mathrm{e}-01$ \\
$0.2+1.5 i$ & 500.31 & $-5 \mathrm{e}-05$ & 0.006 & $9 \mathrm{e}-01$ & $8 \mathrm{e}-02$ & $2 \mathrm{e}-01$ & $3 \mathrm{e}-01$ \\
$0.2+1.5 i$ & 999.79 & $-5 \mathrm{e}-05$ & 0.011 & $1 \mathrm{e}-01$ & $2 \mathrm{e}-02$ & $7 \mathrm{e}-02$ & $1 \mathrm{e}-01$ \\
\hline $0.2+1.8 i$ & 236.52 & $1 \mathrm{e}-04$ & 0.011 & $6 \mathrm{e}-01$ & $6 \mathrm{e}-02$ & $1 \mathrm{e}-01$ & $3 \mathrm{e}-01$ \\
$0.2+1.8 i$ & 500.31 & $2 \mathrm{e}-05$ & 0.004 & $1 \mathrm{e}+00$ & $8 \mathrm{e}-02$ & $2 \mathrm{e}-01$ & $3 \mathrm{e}-01$ \\
$0.2+1.8 i$ & 999.79 & $-4 \mathrm{e}-05$ & 0.007 & $3 \mathrm{e}-01$ & $2 \mathrm{e}-02$ & $6 \mathrm{e}-02$ & $1 \mathrm{e}-01$ \\
\hline
\end{tabular}

TABLE 3. Value statistics of $\operatorname{Im} G_{\frac{1}{2}+i R}(z ; w)$ on $\operatorname{PSL}(2, \mathbb{Z})$ in the box $F$.
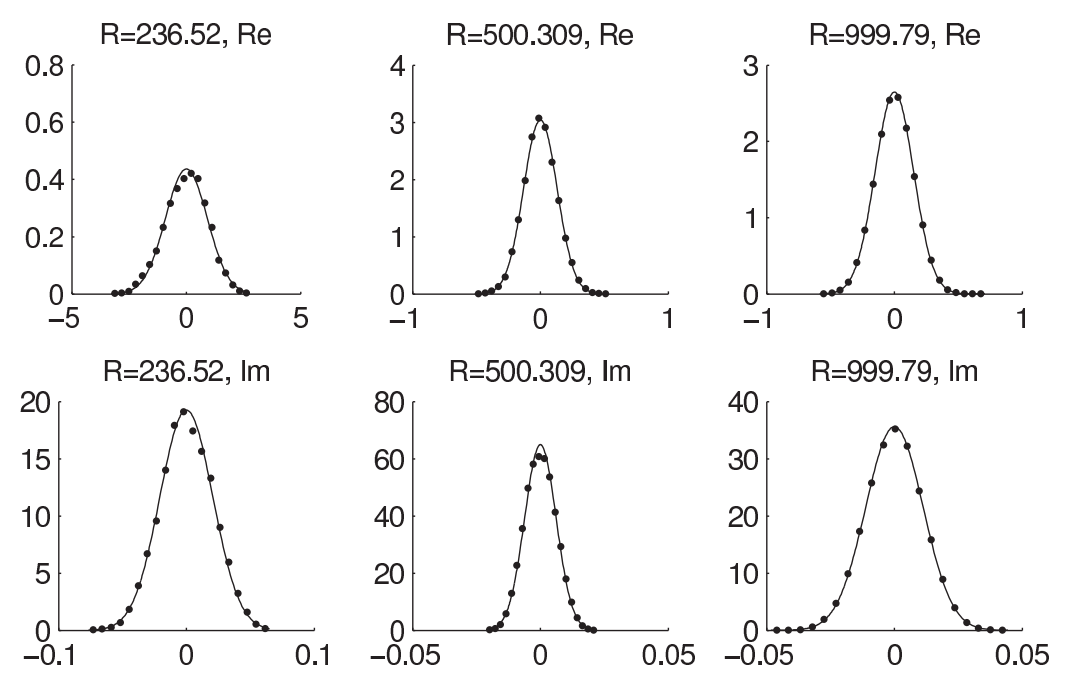

FIGURE 3. Histograms of the real and imaginary parts of $G_{\frac{1}{2}+i R}(z ; 0.2+1.5 i)$, in the first and second rows respectively. The solid lines are Gaussian curves.
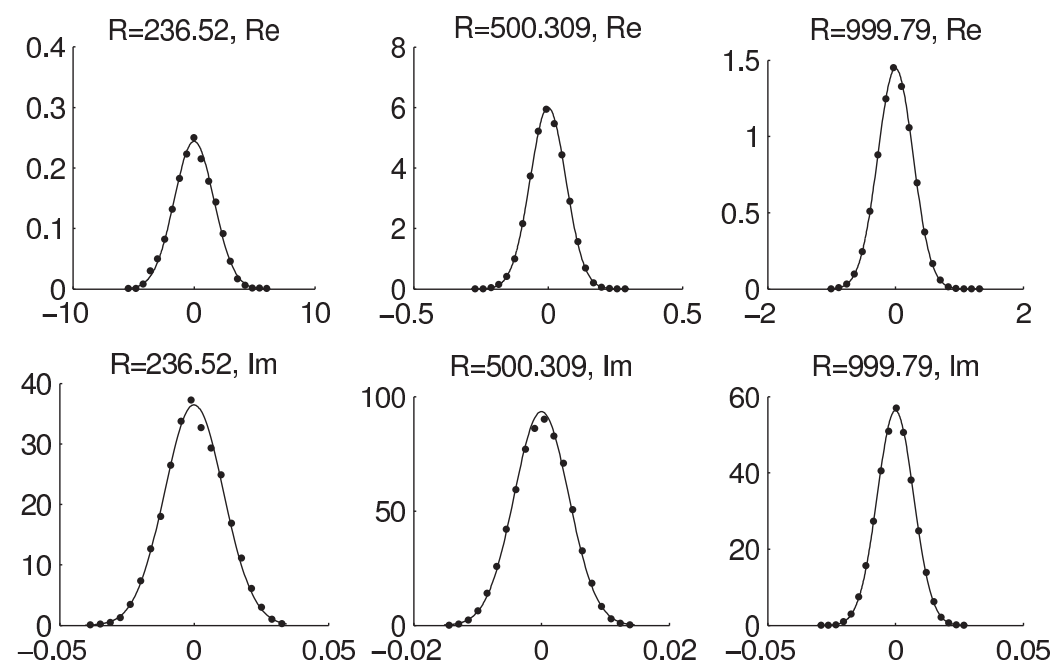

FIGURE 4. Histograms of the real and imaginary parts of $G_{\frac{1}{2}+i R}(z ; 0.2+1.8 i)$, in the first and second rows respectively. The solid lines are Gaussian curves. 
We have also made topography figures of $G_{s}(z ; w)$ in $F$ using the data computed for the statistics tests; see [Avelin 07b].

\section{VALUE STATISTICS FOR $\boldsymbol{F}_{\boldsymbol{n}}(z ; s)$}

The functions $F_{n}(z ; s)$ for $n \neq 0$ can be thought of as generalizations of the Eisenstein series (although they increase much more rapidly at the cusps); cf. Section 1 . With [Avelin 08] in mind, it is thus natural to perform value statistics tests with $F_{n}(z ; s)$. With the basic setup exactly the same as in Section 2, we computed values of $F_{n}(z ; s)$ with $n=1, \ldots, 5$ and $s=1 / 2+i R$ for $R=98.831194 \ldots, R=500$, and $R=1000$. (The fact that the value $s=1 / 2+98.831194 \ldots$ is a zero of the Riemann zeta function has no special significance in this particular test.) Numerical details and sample errors are given in Table 4 . We used working precision $P$ and called the Bessel routines with precision $P_{B}$. The error estimate is computed as in Table 1, but with [Avelin 10, p. 21 (45)] in place of (2-2). See [Avelin 10, Section 3] for the remaining notation $\left(M_{0}, Q, Y\right)$.

Results of our statistical tests are presented in Tables 5 and 6 for the real and imaginary parts of $F_{n}(z ; s)$ respectively, and in Figure 5 for $n=1$ and Figure 6 for $n=5$. The corresponding figures for $n=2,3,4$ are of similar quality, and we present these in [Avelin 07a, Section 3].

Although the improvement of the fit to a Gaussian curve is not as strong as with $E(z ; s)$ in [Avelin 08], our results for $F_{n}(z ; s)$ do suggest that the values of $F_{n}(z ; s)$ have a Gaussian limit distribution as $\operatorname{Im} s \rightarrow \infty$ for $\operatorname{Re} s=1 / 2$.

\begin{tabular}{|cc|ccccc|}
\hline$n$ & $R$ & $M_{0}$ & $Q$ & $P$ & $P_{B}$ & error \\
\hline \hline 1 & 98.83 & 47 & 48 & 38 & 38 & $1 \mathrm{e}-37$ \\
1 & 500.00 & 108 & 147 & 38 & 38 & $1 \mathrm{e}-20$ \\
1 & 1000.00 & 210 & 226 & 97 & 174 & $1 \mathrm{e}-27$ \\
\hline 2 & 98.83 & 55 & 56 & 38 & 38 & $1 \mathrm{e}-36$ \\
2 & 500.00 & 120 & 147 & 38 & 38 & $1 \mathrm{e}-34$ \\
2 & 1000.00 & 225 & 226 & 97 & 174 & $1 \mathrm{e}-37$ \\
\hline 3 & 98.83 & 62 & 63 & 38 & 38 & $1 \mathrm{e}-35$ \\
3 & 500.00 & 129 & 147 & 38 & 38 & $1 \mathrm{e}-35$ \\
3 & 1000.00 & 209 & 230 & 38 & 174 & $1 \mathrm{e}-25$ \\
\hline 4 & 98.83 & 69 & 70 & 38 & 38 & $1 \mathrm{e}-36$ \\
4 & 500.00 & 138 & 147 & 38 & 38 & $1 \mathrm{e}-35$ \\
4 & 1000.00 & 220 & 230 & 38 & 174 & $1 \mathrm{e}-34$ \\
\hline 5 & 98.83 & 76 & 77 & 38 & 38 & $1 \mathrm{e}-36$ \\
5 & 500.00 & 146 & 147 & 38 & 38 & $1 \mathrm{e}-34$ \\
5 & 1000.00 & 229 & 230 & 38 & 174 & $1 \mathrm{e}-34$ \\
\hline
\end{tabular}

TABLE 4. Numerical details of our computations of $F_{n}(z$; $\left.\frac{1}{2}+i R\right)$ on $\operatorname{PSL}(2, \mathbb{Z})$. Our $Y$-values were $0.85,0.86$.
Our remarks about the size of $F$ (here it corresponds to $7 \tau, 35 \tau$, and $71 \tau$ for our three $R$-values respectively), the grid size, and computational time in Section 2 apply to our $F_{n}(z ; s)$-computations as well. One would like to use larger $F$ and finer grid size also for $F_{n}(z ; s)$, at least for a few examples, to gain better accuracy in the $I_{k}$.

We have made topographical figures of $F_{n}(z ; s)$ in $F$ using the data computed for these statistics tests; see [Avelin 07b].

\section{FURTHER EXPERIMENTS}

\subsection{Nonarithmetic Groups}

Of course, one expects to find similar Gaussian behavior for $G_{s}(z ; w)$ and $F_{n}(z ; s)$ when the group $\Gamma$ is nonarithmetic, i.e., a natural counterpart of what we found for the Eisenstein series in [Avelin 08].

\subsection{Computing Higher Fourier Coefficients of $F_{n}(z ; s)$}

Statistical studies of the Fourier coefficients of cusp forms and Eisenstein series are presented in [Hejhal 99] and [Avelin 08]. In order to perform similar tests on the Fourier coefficients of $F_{n}(z ; s)$, one needs to be able to compute $\varphi_{m}^{n}(s)$ for large $m$; cf. (1-4). The ideas in [Hejhal 99, p. 299] for Fourier coefficients of cusp forms are also applicable to $F_{n}(z ; s)$. However, these ideas require the computations of a sum involving values of $F_{n}\left(z_{j}^{*} ; s\right)$, where some $\operatorname{Im} z_{j}^{*}$ are very large. This sum suffers catastrophic cancellation due to the $I$-Bessel term in (1-4). Therefore the method in [Hejhal 99, p. 299] applied to $F_{n}(z ; s)$ requires $I$-Bessel computations of very large precision. With our present methods for the $I$ Bessel function, cf. [Avelin 10, Section 5], we are unable to achieve this. But the high-precision computations of the $K$-Bessel function in [Booker et al. 06] offer a promising approach also for the $I$-Bessel function. We hope to return to this in a later publication.

\subsection{On the Appearance of $G_{s}(z ; w)$ in Cusp Form Computations}

In [Hejhal 92], the author used a version of his cusp form algorithm to search for pseudo cusp forms on $\operatorname{PSL}(2, \mathbb{Z})$ and other Hecke triangle groups; cf. [Hejhal 81, p. 99]. Hejhal's algorithm computes eigenfunctions $\Phi(z)$ of the Laplacian with a Fourier expansion of the type

$$
\Phi(z)=\sum_{n=1}^{\infty} c_{n} y^{1 / 2} K_{s-\frac{1}{2}}(2 \pi|n| y)\left\{\begin{array}{l}
\cos (2 \pi n x), \\
\sin (2 \pi n x),
\end{array}\right.
$$

where the factor $\cos (2 \pi n x)$ or $\sin (2 \pi n x)$ reflects that $\Phi(z)$ is either even or odd with respect to the reflection 


\begin{tabular}{|cc|cccccc|}
\hline$n$ & $s$ & mean & $\sigma$ & $\delta$ & $I_{4}$ & $I_{6}$ & $I_{8}$ \\
\hline \hline 1 & 98.83 & $2 \mathrm{e}-03$ & 0.19 & $6 \mathrm{e}-02$ & $-5 \mathrm{e}-02$ & $-5 \mathrm{e}-02$ & $4 \mathrm{e}-02$ \\
1 & 500.00 & $7 \mathrm{e}-05$ & 0.07 & $4 \mathrm{e}-02$ & $-7 \mathrm{e}-03$ & $-3 \mathrm{e}-02$ & $-5 \mathrm{e}-02$ \\
1 & 1000.00 & $5 \mathrm{e}-05$ & 0.05 & $4 \mathrm{e}-02$ & $2 \mathrm{e}-02$ & $5 \mathrm{e}-02$ & $9 \mathrm{e}-02$ \\
\hline 2 & 98.83 & $-7 \mathrm{e}-04$ & 0.09 & $2 \mathrm{e}-01$ & $2 \mathrm{e}-01$ & $4 \mathrm{e}-01$ & $5 \mathrm{e}-01$ \\
2 & 500.00 & $-4 \mathrm{e}-05$ & 0.04 & $8 \mathrm{e}-02$ & $3 \mathrm{e}-02$ & $7 \mathrm{e}-02$ & $1 \mathrm{e}-01$ \\
2 & 1000.00 & $-2 \mathrm{e}-05$ & 0.04 & $5 \mathrm{e}-02$ & $2 \mathrm{e}-02$ & $7 \mathrm{e}-02$ & $1 \mathrm{e}-01$ \\
\hline 3 & 98.83 & $-2 \mathrm{e}-03$ & 0.24 & $6 \mathrm{e}-02$ & $1 \mathrm{e}-02$ & $9 \mathrm{e}-02$ & $2 \mathrm{e}-01$ \\
3 & 500.00 & $-5 \mathrm{e}-05$ & 0.11 & $2 \mathrm{e}-02$ & $3 \mathrm{e}-02$ & $9 \mathrm{e}-02$ & $2 \mathrm{e}-01$ \\
3 & 1000.00 & $-4 \mathrm{e}-05$ & 0.05 & $4 \mathrm{e}-02$ & $2 \mathrm{e}-02$ & $3 \mathrm{e}-02$ & $3 \mathrm{e}-02$ \\
\hline 4 & 98.83 & $-3 \mathrm{e}-03$ & 0.20 & $1 \mathrm{e}-01$ & $2 \mathrm{e}-01$ & $4 \mathrm{e}-01$ & $6 \mathrm{e}-01$ \\
4 & 500.00 & $3 \mathrm{e}-05$ & 0.07 & $4 \mathrm{e}-02$ & $3 \mathrm{e}-02$ & $6 \mathrm{e}-02$ & $1 \mathrm{e}-01$ \\
4 & 1000.00 & $-4 \mathrm{e}-05$ & 0.05 & $3 \mathrm{e}-02$ & $2 \mathrm{e}-03$ & $4 \mathrm{e}-03$ & $5 \mathrm{e}-03$ \\
\hline 5 & 98.83 & $2 \mathrm{e}-03$ & 0.25 & $8 \mathrm{e}-02$ & $6 \mathrm{e}-02$ & $2 \mathrm{e}-01$ & $4 \mathrm{e}-01$ \\
5 & 500.00 & $1 \mathrm{e}-04$ & 0.11 & $3 \mathrm{e}-02$ & $2 \mathrm{e}-02$ & $7 \mathrm{e}-02$ & $2 \mathrm{e}-01$ \\
5 & 1000.00 & $-8 \mathrm{e}-06$ & 0.05 & $4 \mathrm{e}-02$ & $2 \mathrm{e}-02$ & $6 \mathrm{e}-02$ & $1 \mathrm{e}-01$ \\
\hline
\end{tabular}

TABLE 5. Value statistics of $\operatorname{Re} F_{n}(z ; 1 / 2+i R)$ on $\operatorname{PSL}(2, \mathbb{Z})$.

\begin{tabular}{|cc|cccccc|}
\hline$n$ & $R$ & mean & $\sigma$ & $\delta$ & $I_{4}$ & $I_{6}$ & $I_{8}$ \\
\hline \hline 1 & 98.83 & $6 \mathrm{e}-03$ & 0.10 & $2 \mathrm{e}-01$ & $1 \mathrm{e}-01$ & $3 \mathrm{e}-01$ & $4 \mathrm{e}-01$ \\
1 & 500.00 & $8 \mathrm{e}-04$ & 0.22 & $2 \mathrm{e}-02$ & $3 \mathrm{e}-02$ & $1 \mathrm{e}-01$ & $2 \mathrm{e}-01$ \\
1 & 1000.00 & $-3 \mathrm{e}-04$ & 0.18 & $8 \mathrm{e}-03$ & $8 \mathrm{e}-03$ & $3 \mathrm{e}-02$ & $6 \mathrm{e}-02$ \\
\hline 2 & 98.83 & $6 \mathrm{e}-03$ & 0.12 & $1 \mathrm{e}-01$ & $-6 \mathrm{e}-02$ & $-4 \mathrm{e}-02$ & $8 \mathrm{e}-02$ \\
2 & 500.00 & $5 \mathrm{e}-04$ & 0.14 & $2 \mathrm{e}-02$ & $2 \mathrm{e}-02$ & $8 \mathrm{e}-02$ & $2 \mathrm{e}-01$ \\
2 & 1000.00 & $3 \mathrm{e}-04$ & 0.28 & $4 \mathrm{e}-03$ & $9 \mathrm{e}-03$ & $3 \mathrm{e}-02$ & $7 \mathrm{e}-02$ \\
\hline 3 & 98.83 & $-1 \mathrm{e}-02$ & 0.21 & $1 \mathrm{e}-01$ & $-7 \mathrm{e}-02$ & $-8 \mathrm{e}-02$ & $2 \mathrm{e}-02$ \\
3 & 500.00 & $-3 \mathrm{e}-04$ & 0.29 & $6 \mathrm{e}-03$ & $2 \mathrm{e}-02$ & $7 \mathrm{e}-02$ & $2 \mathrm{e}-01$ \\
3 & 1000.00 & $2 \mathrm{e}-05$ & 0.13 & $1 \mathrm{e}-02$ & $6 \mathrm{e}-03$ & $3 \mathrm{e}-02$ & $1 \mathrm{e}-01$ \\
\hline 4 & 98.83 & $-2 \mathrm{e}-03$ & 0.09 & $2 \mathrm{e}-01$ & $7 \mathrm{e}-02$ & $2 \mathrm{e}-01$ & $4 \mathrm{e}-01$ \\
4 & 500.00 & $-5 \mathrm{e}-04$ & 0.16 & $2 \mathrm{e}-02$ & $3 \mathrm{e}-02$ & $1 \mathrm{e}-01$ & $2 \mathrm{e}-01$ \\
4 & 1000.00 & $-3 \mathrm{e}-04$ & 0.32 & $4 \mathrm{e}-03$ & $1 \mathrm{e}-02$ & $4 \mathrm{e}-02$ & $8 \mathrm{e}-02$ \\
\hline 5 & 98.83 & $-2 \mathrm{e}-03$ & 0.07 & $3 \mathrm{e}-01$ & $-1 \mathrm{e}-01$ & $-2 \mathrm{e}-01$ & $-2 \mathrm{e}-01$ \\
5 & 500.00 & $-3 \mathrm{e}-04$ & 0.32 & $7 \mathrm{e}-03$ & $4 \mathrm{e}-03$ & $3 \mathrm{e}-02$ & $1 \mathrm{e}-01$ \\
5 & 1000.00 & $-4 \mathrm{e}-04$ & 0.33 & $4 \mathrm{e}-03$ & $2 \mathrm{e}-02$ & $7 \mathrm{e}-02$ & $1 \mathrm{e}-01$ \\
\hline
\end{tabular}

TABLE 6. Value statistics of $\operatorname{Im} F_{n}(z ; 1 / 2+i R)$ on $\operatorname{PSL}(2, \mathbb{Z})$.
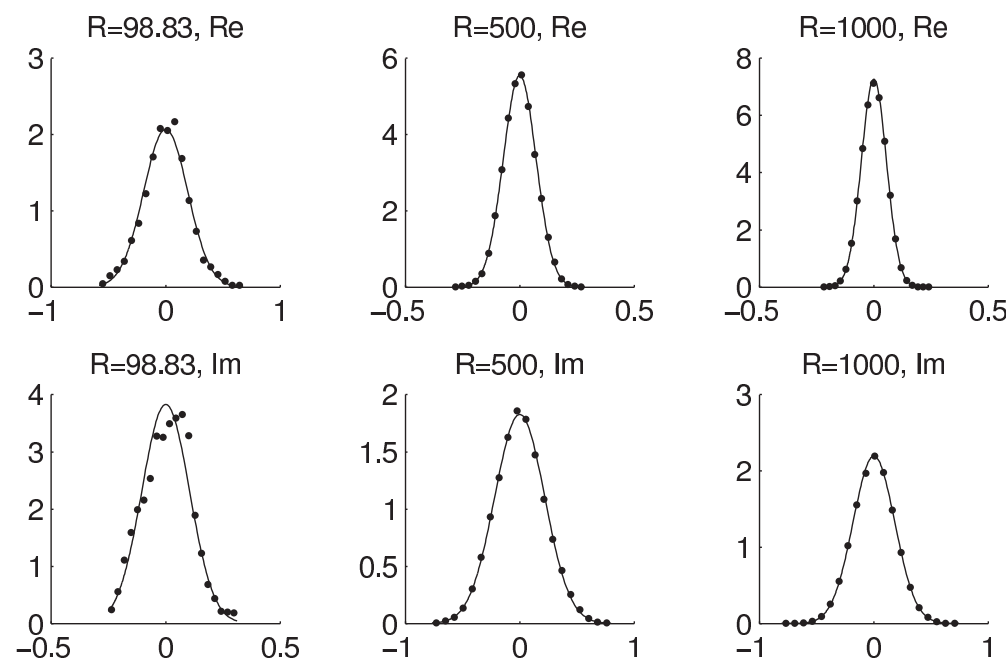

FIGURE 5. Histograms of the real and imaginary parts of $F_{1}(z ; 1 / 2+i R)$, in the first and second rows respectively. The solid lines are Gaussian curves. 

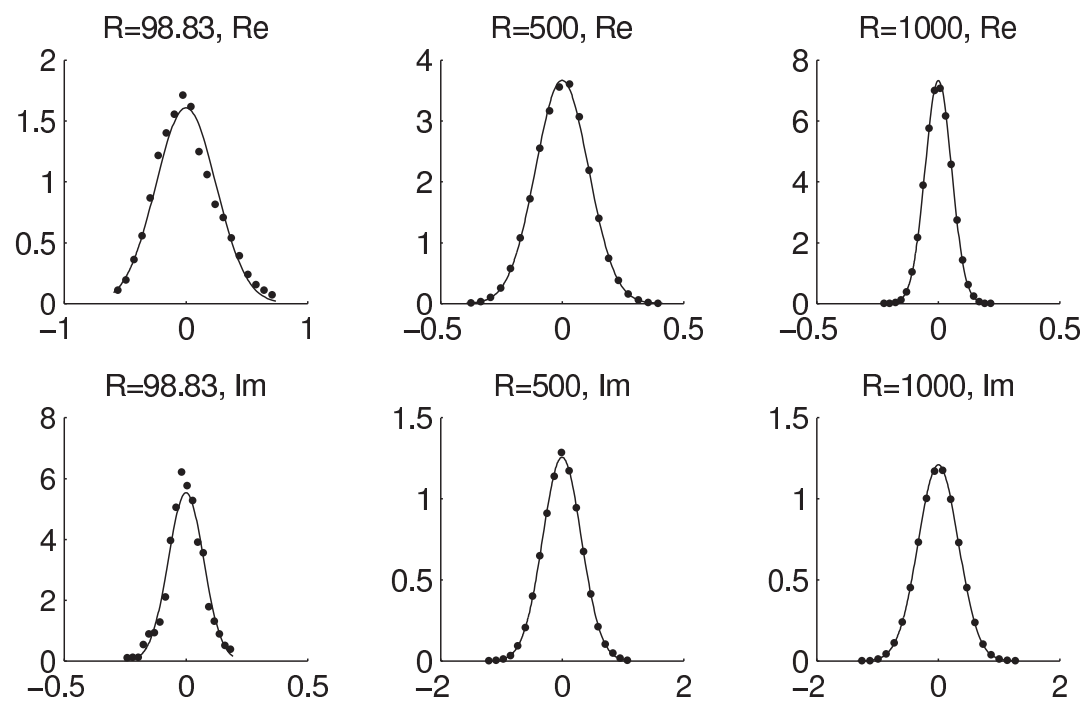

FIGURE 6. Histograms of the real and imaginary parts of $F_{5}(z ; 1 / 2+i R)$, in the first and second rows respectively. The solid lines are Gaussian curves.

$\mathcal{R} z=-\bar{z}$. The Fourier coefficients $c_{n}$ are required to be real and normalized with $c_{1}=1$.

At first glance, the functions computed in [Hejhal 92] all seem to be of the form

$$
G_{s}(z ; \rho) \quad \text { with } \quad E(\rho ; s)=0
$$

where $E(\rho ; s)$ is the Eisenstein series; cf. (1-1) and (1-2) or [Hejhal 92, p. 45]. On $\operatorname{PSL}(2, \mathbb{Z})$, for example, Hejhal was able to detect $G_{s}(z ; \rho)$, with $s$ the first zero of the Riemann zeta function, i.e., $s=1 / 2+i 14.134725 \ldots$; cf. [Hejhal 92, Section 8] and [Avelin 10, Table 6]. The fact that no other $G_{s}(z ; w)$ seem to appear is somewhat puzzling, considering that for each $w$ sufficiently near $\rho$ there exists (by elementary complex variables) some $s=$ $1 / 2+i R$ with $R$ near $14.134 \ldots$ such that $E(w ; s)=0$.

It might be possible to use our $G_{s}(z ; w)$-algorithm to explore the reason that the functions $G_{s}(z ; \rho)$ with $E(\rho ; s)=0$ seem to stand out so strongly in Hejhal's experiments. It appears that the fact that $\rho$ is an elliptic fixed point of order three plays a central role in this explanation. For example, the nodal curves of the Eisenstein series through $\rho$ can be visualized to leading order for $R \approx R_{0}$ and $z \approx \rho$ by expanding $E(z ; s)$ into a Taylor series about $s=s_{0}$ and then using Taylor series representations for $E\left(z ; 1 / 2+i R_{0}\right)$ and $E_{s}\left(z ; 1 / 2+i R_{0}\right)$ about $z=\rho$; cf. [Hejhal 92, p. 93, (9.2)] and [Avelin 07a, (16)]. One finds that the curve along which $E(z ; 1 / 2+i R)$ is zero intersects $\partial \mathcal{F}$ approximately at distance $C\left(R-R_{0}\right)^{1 / 3}$ from $\rho$, for some constant $C$; that is, this intersection is relatively distant from $\rho$. The power $1 / 3$ is a direct consequence of $\rho$ being of order three.

Another consequence of the fact that $\rho$ is an elliptic fixed point of order three seems to be that nodal curves for the functions

$$
f_{n}(w)=\frac{\operatorname{Re} F_{-n}(w ; s)}{\operatorname{Re} F_{-1}(w ; s)}-\frac{\operatorname{Re} F_{-n}\left(\rho ; s_{0}\right)}{\operatorname{Re} F_{-1}\left(\rho ; s_{0}\right)}
$$

(which are related to the normalized Fourier coefficients of pseudo cusp forms) are much closer together for $s=$ $1 / 2+i R_{0}$ than the corresponding curves for other $s=$ $1 / 2+i R$ with $R$ near $R_{0}$; cf. [Avelin 07a, Section 4], especially (17) and the paragraph following Table 9.

Although further investigation is necessary to provide a complete explanation, these circumstances are likely to be part of it. See [Avelin 07a, Section 4] for some additional insights.

\section{ACKNOWLEDGMENTS}

The author is grateful to Dennis Hejhal and Andreas Strömbergsson for helpful discussions on this work.

\section{REFERENCES}

[Avelin 07a] H. Avelin. "Computations of the Green's Function and Its Fourier Coefficients on $\operatorname{PSL}(2, \mathbb{Z}) . "$ Available online (http://www.math.uu.se/research/pub/ Avelin5.pdf), 2007.

[Avelin 07b] H. Avelin. "Topography of the Green's Function and Its Fourier Coefficients on $\operatorname{PSL}(2, \mathbb{Z})$." Available online (http://www.math.uu.se/research/archive/avelin/ greendata/Topography_Green.pdf), 2007. 
[Avelin 08] H. Avelin. "Computations of Eisenstein Series on Fuchsian Groups." Math. Comp. 77 (2008), 1779-1800.

[Avelin 10] H. Avelin. "Computations of Green's Function and Its Fourier Coefficients on Fuchsian Groups." Exp. Math. 19:3 (2010), 317-334.

[Booker et al. 06] A. Booker, A. Strömbergsson, and A. Venkatesh. "Effective Computations of Maass Cusp Forms." Int. Math. Res. Not. (2006), 34pp., Art. ID 71281.

[de Vries et al. 98] P. de Vries, D. V. van Coevorden, and A. Lagendijk. "Point Scatterers for Classical Waves." Reviews of Modern Physics 70:2 (1998), 447-466.

[Hejhal 81] D. A. Hejhal. "Some Observations Concerning Eigenvalues of the Laplacian and Dirichlet $L$-Series." In Recent Progress in Analytic Number Theory, vol. 2, pp. 95110. New York: Academic Press, 1981.

[Hejhal 83] D. A. Hejhal. The Selberg Trace Formula for $\operatorname{PSL}(2, \mathbb{R})$, vol. 2, Lecture Notes in Math. 1001. Berlin: Springer, 1983.

[Hejhal 92] D. A. Hejhal. Eigenvalues of the Laplacian for Hecke Triangle Groups, Mem. Amer. Math. Soc. 469. Providence: AMS, 1992.
[Hejhal 99] D. A. Hejhal. "On Eigenfunctions of the Laplacian for Hecke Triangle Groups." In Emerging Applications of Number Theory, edited by D. Hejhal, J. Friedman, M. Gutzwiller, and A. Odlyzko, pp. 291-315. New York: Springer, 1999.

[Hejhal and Rackner 92] D. A. Hejhal and B. N. Rackner. "On the Topography of Maass Waveforms for $\operatorname{PSL}(2, \mathbb{Z}) . "$ Exp. Math. 1:4 (1992), 275-305.

[Hejhal and Strömbergsson 01] D. A. Hejhal and A. Strömbergsson. "On Quantum Chaos and Maass Waveforms of CM-Type." Found. Phys. 31:3 (2001), 519-533.

[Šeba 90] P. Šeba. "Wave Chaos in Singular Quantum Billiard." Physical Review Letters 64:16 (1990), 1855-1858.

[Shigehara 94] T. Shigehara. "Conditions for the Appearance of Wave Chaos in Quantum Singular Systems with a Pointlike Scatterer." Phys. Rev. E 50 (1994), 4357-4370.

[Shigehara and Cheon 97] T. Shigehara and T. Cheon. "Spectral Properties of Three-Dimensional Quantum Billiards with a Pointlike Scatterer." Phys. Rev. E 55 (1997), $6832-6844$

Helen Avelin, Department of Mathematics, Uppsala University, S-751 06, Uppsala, Sweden (helen.avelin@gmail.com)

Received September 27, 2007; accepted in revised form August 25, 2009. 